Canadian

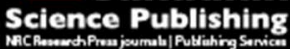

Applied Physiology, Nutrition, and Metabolism Physiologie appliquée, nutrition et métabolisme

\title{
Short-term high-fat diet alters postprandial glucose metabolism and circulating vascular cell adhesion molecule- 1 in healthy males
}

\begin{tabular}{|r|l|}
\hline Journal: & Applied Physiology, Nutrition, and Metabolism \\
\hline Manuscript ID & apnm-2015-0702.R2 \\
\hline Manuscript Type: & Article \\
\hline Date Submitted by the Author: & O4-Apr-2016 \\
\hline Complete List of Authors: & $\begin{array}{l}\text { Numao, Shigeharu; Kyoto Pharmaceutical University } \\
\text { Kawano, Hiroshi; Kokushikan University } \\
\text { Endo, Naoya; Japan Institute of Sports Sciences } \\
\text { Yamada, Yuka; Waseda Institute for Sport Sciences } \\
\text { Takahashi, Masaki; Waseda University, Faculty of Science and Engineering } \\
\text { Konishi, Masayuki; Waseda University, Faculty of Sport Sciences } \\
\text { Sakamoto, Shizuo; WASEDA UNIVERSITY, FACULTY OF SPORT SCIENCES }\end{array}$ \\
\hline Keyword: & $\begin{array}{l}\text { first-phase insulin release, insulinogenic index, meal tolerance test, } \\
\text { glucagon-like peptide-1, low-carbohydrate/high-fat diet }\end{array}$ \\
\hline &
\end{tabular}




\section{Title}

Short-term high-fat diet alters postprandial glucose metabolism and circulating vascular cell adhesion molecule- 1 in healthy males

\section{Author names}

Shigeharu Numao ${ }^{\mathrm{a}, \mathrm{b}}$, Hiroshi Kawano ${ }^{\mathrm{c}}$, Naoya Endo ${ }^{\mathrm{d}}$, Yuka Yamada ${ }^{\mathrm{b}}$, Masaki Takahashi ${ }^{\mathrm{e}}$, Masayuki Konishi ${ }^{\mathrm{f}}$, Shizuo Sakamoto ${ }^{\mathrm{f}}$

\section{Corresponding author}

Shigeharu Numao

Department of Health and Sports Sciences, Kyoto Pharmaceutical University

Postal address: 5 Nakauchi-cho, Misasagi, Yamashina-ku, Kyoto 607-8414, JAPAN

Phone: +81-75-595-4675

E-mail: numashige@mb.kyoto-phu.ac.jp

\section{Author affiliations}

a Department of Health and Sports Sciences, Kyoto Pharmaceutical University

5 Nakauchi-cho, Misasagi, Yamashina-ku, Kyoto 607-8414, JAPAN

${ }^{\mathrm{b}}$ Waseda Institute for Sport Sciences

2-579-15 Mikajima, Tokorozawa, Saitama 359-1192, JAPAN

${ }^{c}$ Faculty of Letters, Kokushikan University

4-28-1 Setagaya, Setagaya-ku, Tokyo 154-8515, JAPAN

dJapan Institute of Sports Sciences

3-15-1 Nishigaoka, Kita-ku, Tokyo 115-0056

${ }^{\mathrm{e}}$ Faculty of Science and Engineering, Waseda University

2-2 Wakamatsu-cho, Shinjuku, Tokyo 162-8480, JAPAN

${ }^{\mathrm{f}}$ Faculty of Sport Sciences, Waseda University

2-579-15 Mikajima, Tokorozawa, Saitama 359-1192, JAPAN 


\begin{abstract}
Short-term intake of a high-fat diet aggravates postprandial glucose metabolism; however, the dose-response relationship has not been investigated. We hypothesized that short-intake of a eucaloric low-carbohydrate/high-fat diet (LCHF) would aggravate postprandial glucose metabolism and circulating adhesion molecules in healthy males. Seven healthy young males (mean \pm SE age: $26 \pm 1$ years) consumed either a eucaloric control diet (C, approximately $25 \%$ fats), a eucaloric intermediate-carbohydrate/intermediate-fat diet (ICIF, approximately $50 \%$ fats), or a LCHF (approximately 70\% fats) for 3 days. An oral meal tolerance test (MTT) was performed after the 3-day dietary intervention. The concentrations of plasma glucose, insulin, glucagon-like peptide-1 (GLP-1), intercellular adhesion molecule-1 (ICAM-1), and vascular cell adhesion molecule-1 (VCAM-1) were determined at rest and during MTT. The incremental area under the curve (iAUC) of plasma glucose concentration during MTT was significantly higher in LCHF than in C $(P=0.009)$. The first-phase insulin secretion indexes were significantly lower in LCHF than in $\mathrm{C}(P=0.04)$. Moreover, the iAUC of GLP-1 and VCAM-1 concentrations was significantly higher in LCHF than in C ( $P$ $=0.014$ and $P=0.04$, respectively). The metabolites from ICIF and C were not significantly different. In conclusion, short-term intake of eucaloric diet containing a high percentage of fats in healthy males excessively increased postprandial glucose and VCAM-1 concentrations and attenuated first-phase insulin release.
\end{abstract}

Keywords: first-phase insulin release, insulinogenic index, meal tolerance test, glucagon-like peptide-1, low-carbohydrate/high-fat diet 


\section{Introduction}

Postprandial hyperglycemia is an independent risk factor for cardiovascular disease morbidity and mortality (Cavalot et al. 2011; The DECODE group 1999, 2001; Nakagami and DECODA Study Group 2004). Even acute postprandial hyperglycemia and acute fluctuation in blood glucose concentration induce vascular endothelial dysfunction (Kawano et al. 1999; Williams et al. 1998), platelet activation (Sakamoto et al. 2000), oxidative stress, and inflammation (Esposito et al. 2002). Therefore, it is very important to elucidate the causes of postprandial hyperglycemia to prevent cardiovascular diseases.

Postprandial hyperglycemia would depend on the content of the diet; specifically, an acute increase in blood glucose concentration may be induced by short-term intake of a eucaloric low-carbohydrate/high-fat diet (LCHF, $>70 \%$ fats). Several studies using an oral glucose tolerance test (OGTT) in non-diabetic individuals have demonstrated that short-term eucaloric LCHF leads to an excessive increase in postprandial glucose concentration and aggravation of postprandial glucose metabolism. Pehleman et al. (2005) reported that compared with a control diet, a 2-day intake of LCHF resulted in a greater increase in postprandial blood glucose and insulin concentrations. Moreover, Numao et al. $(2012,2013)$ demonstrated that compared with a control diet, a 3-day intake of LCHF resulted in a greater 
increase in postprandial blood glucose concentration and decrease in first-phase insulin secretion. Despite these findings, the dose-response relationship of short-term intake of carbohydrates and fats with aggravation of postprandial glucose metabolism has not been investigated.

Atherosclerosis is usually initiated by endothelial activation that releases soluble forms of adhesion molecules (Mah and Bruno 2012). Therefore, some circulating soluble adhesion molecules, such as intercellular adhesion molecule-1 (ICAM-1) and vascular cell adhesion molecule-1 (VCAM-1), can be biomarkers of endothelial dysfunction. In healthy individuals as well as in diabetic patients, circulating ICAM-1 and VCAM-1 concentrations have been reported to increase during hyperglycemia (Ceriello et al. 2004; Derosa et al. 2010; Marfella et al. 2000). This suggests that hyperglycemia may elicit endothelial activation and development of atherosclerosis. However, the effects of aggravation of postprandial glucose metabolism induced by short-term dietary fat intake on circulating adhesion molecules have been unclear.

The purpose of this study was to determine the effects of short-term dietary fat content on postprandial glucose metabolism and circulating adhesion molecules in healthy males. 


\section{Methods and materials}

\subsection{Participants}

Seven healthy non-diabetic young males (age: $26 \pm 1$ years; height: $179 \pm 1 \mathrm{~cm}$; fasting glucose concentration: $<126 \mathrm{mg} / \mathrm{dl}$ ) participated in this study. We believe that males are more susceptible to diet-related alterations in postprandial glucose metabolism, because of higher incidences of diabetes and impaired glucose tolerance in males than in females (Cowie et al. 2009; Hennninger et al. 2015; Soriguer et al. 2012). Because of the clinical implications, we recruited only males as participants in the present study. None of the participants had regular exercise habits and history of any metabolic and cardiovascular diseases. All participants were non-smokers and were not on any medication or supplements. The purpose, design, and risks of this study were explained to all participants and each provided written informed consent. The study conformed to the principles outlined in the Helsinki Declaration and was approved by the ethics committee of Waseda University (2011-207).

\subsection{Study protocol}

Three experimental trials lasting 4 days each were administered to participants in a crossover design. These trials consisted of a 3-day intake of a control diet (C), intermediate-carbohydrate/intermediate-fat diet (ICIF), or LCHF. The order of the trials was 
randomized in each participant. The wash-out period was set at $>1$ week. One day before each trial, the participants were instructed to eat a uniform diet and to take their evening meal before 21:00; thereafter, they were asked to refrain from consuming any food except water. The next morning (day 1), the participants arrived at the laboratory at 09:00 after an overnight fast $(>12 \mathrm{~h})$. After a 30 -min rest period in the supine position, blood sample was taken to measure baseline metabolites and hormone concentrations. In addition, body mass, fat mass (FM), and fat-free mass (FFM) were measured using the impedance method (InnerScan BC-520; Tanita, Tokyo, Japan). After these measurements, the participants consumed the designated trial diets until day 3 (for 3 days).

On day 4 (after the 3-day dietary intervention) of each trial, the participants arrived at the laboratory at 09:00 after an overnight fast. Initially, body mass, FM, and FFM were measured using the impedance method (InnerScan BC-520). After a 30-min rest period in the supine position, an intravenous catheter was inserted into the antecubital vein and a blood sample was obtained. Thereafter, a 120-min meal tolerance test (MTT) was started using an experimental meal (JANEF E460F18; Kewpie Corporation, Tokyo, Japan). The experimental meal for MMT was composed of cream chicken, cracker, and pudding, with $460 \mathrm{kcal}$ energy, $18.0 \mathrm{~g}$ protein, $18.0 \mathrm{~g}$ fats, and $56.5 \mathrm{~g}$ carbohydrates. During MTT, the participants rested 
supine on a bed while blood samples were drawn at 15, 30, 45, 60, 90, and 120 min after consuming the experimental meal.

\subsection{Experimental diets}

The participants consumed the 3 experimental diets (C, ICIF, and LCHF) for 3 consecutive days each. The menu of these diets was prepared by a skilled nutritionist using a dietary analysis program (Eiyoukun, version 4.0; Kenpakusya, Tokyo, Japan) and was provided to the participants by the investigators. The total energy intake per day of each participant was calculated on the basis of the equation established by the Dietary Reference Intakes for Japanese in 2010: [basal metabolic rate (reference basal metabolic rate $\times$ reference body mass) $\times$ physical activity level (normal physical activity level, 1.75)] (Ministry of Health Labour and Welfare 2010). The total energy intake per day was similar among the 3 experimental diets, whereas the percentage of fats and carbohydrates to total energy intake varied. The approximate contents of $\mathrm{C}$ were $10 \%$ protein, $30 \%$ fats, and $60 \%$ carbohydrates; those of ICIF were $10 \%$ protein, $50 \%$ fats, and $40 \%$ carbohydrates; and those of LCHF were $10 \%$ protein, $70 \%$ fats, and $20 \%$ carbohydrates (Table 1). Based on our previous studies

(Numao et al. 2012, 2013) and other previous study (Pehleman et al. 2005), the percentage of protein, fats, and carbohydrates in each experimental diet was determined as follows; first, C 
was set as the recommended diet with recommended quantity of nutrients (Ministry of Health Labour and Welfare, 2010). Second, although the percentage of fat is extremely high, LCHF was set as the diet containing the percentage of fat (approximately 70\%) that is likely to aggravate postprandial glucose metabolism, as seen in previous studies (Numao et al. 2012, 2013; Pehleman et al. 2005). Finally, ICIF was set as the diet containing the percentage of fat that was intermediate between that of $\mathrm{C}$ and LCHF so that the dose-response relationship between percentage of fat intake and postprandial glucose metabolism could be determined within the range of $\mathrm{C}$ to LCHF. These diets were constant every day and were designed to be as common and as regular as possible. The participants were instructed to adhere to the provided menu, to check the food intake record, and to maintain their usual lifestyle and physical activities throughout the experiment.

\subsection{Blood sampling and analysis}

For analysis of glucose concentration, blood samples were collected in a 2-mL tube containing sodium fluoride, heparin sodium, and disodium ethylenediaminetetraacetate (EDTA-2Na). For analysis of insulin, free fatty acid (FFA), $\beta$-hydroxybutyric acid ( $\beta$-HBA), triglyceride (TG), total cholesterol (TC), high-density lipoprotein cholesterol (HDLC), alanine aminotransferase (ALT), aspartate aminotransferase (AST), ICAM-1, and VCAM-1 
concentrations, blood samples were collected in an 8-mL tube containing thrombin and silica.

For analysis of glucose-dependent insulinotropic polypeptide (GIP) and glucagon-like peptide-1 (GLP-1) concentrations, blood samples were collected in a 2-mL tube containing $2.5 \mathrm{mg}$ EDTA, $1000 \mathrm{KIU}$ aprotinin, and $20 \mu \mathrm{l}$ dipeptidyl peptidase-4 inhibitor (Millipore, MA, USA). Blood samples in 2-mL tubes were kept on ice and centrifuged at $3000 \times g$ for 10 min at $4^{\circ} \mathrm{C}$ within 30 min of collection; samples in 8 -mL tubes were centrifuged at $3000 \times \mathrm{g}$ for $10 \mathrm{~min}$ at $4^{\circ} \mathrm{C}$ after $30 \mathrm{~min}$ of collection. After centrifugation, the plasma and serum obtained from each sample were transferred into plastic tubes and immediately stored at $-80^{\circ} \mathrm{C}$ until further analysis.

Plasma glucose concentration was analyzed using an enzymatic colorimetric method [Wako Pure Chemical Industries, Osaka, Japan; intra-assay coefficient of variation $(\mathrm{CV}),<0.22 \%]$.

Serum insulin concentration was measured using electrochemiluminescence immunoassay (Roche Diagnostics, Tokyo, Japan; intra-assay CV: $<1.3 \%$ ). Serum FFA concentration was analyzed using an enzymatic colorimetric method (Wako Pure Chemical Industries; intra-assay $\mathrm{CV}:<0.39 \%$ ). Serum $\beta$-HBA concentration was measured by an enzymatic cycling method (Kainos Laboratories, Tokyo, Japan; intra-assay CV: $<0.55 \%$ ). Serum TG concentration was analyzed using an enzymatic colorimetric method with a glycerol blank 
sample (Kyowa Medex, Tokyo, Japan; intra-assay CV, $<0.82 \%$ ). Serum TC and HDLC concentrations were measured by an enzymatic method (Kyowa Medex; intra-assay CV: $<0.92 \%$ and $<0.52 \%$, respectively). The concentration of low-density lipoprotein cholesterol (LDLC) was calculated according to the equation of Friedewald et al. (1972). Serum ALT and AST concentrations were determined by the Japan Society of Clinical Chemistry transferable method (Wako Pure Chemical Industries; intra-assay CV: $<0.54 \%$ and $<1.87 \%$, respectively). Serum ICAM-1 and VCAM-1 and plasma GIP and GLP-1 (7-36 and 7-37 amides) concentrations were measured by enzyme-linked immunosorbent assays (Millipore; intra-assay $\mathrm{CV}:<6.7 \%,<6.7 \%,<8.8 \%$, and $<8.0 \%$, respectively). To eliminate inter-assay variation, samples from each participant were analyzed in the same run.

\subsection{Assessment of metabolites, insulin sensitivity, and insulin secretion}

Changes in the concentrations of metabolites (glucose, insulin, FFA, $\beta$-HBA, TG, GIP, GLP-1, ICAM-1, and VCAM-1) during the 120-min MTT were assessed by the incremental area under the curve (iAUC), which was calculated by the trapezoidal rule. Insulin sensitivity was assessed by the homeostasis model assessment index of insulin resistance (HOMA-IR), Matsuda index (Matsuda and DeFronzo 1999), and oral glucose insulin sensitivity (OGIS) (Mari et al. 2001); first-phase insulin release was assessed by the insulinogenic and Stumvoll 
index (Stumvoll et al. 2000).

\subsection{Statistical analysis}

All data are represented as mean \pm standard error (SE) and were tested for normality using the Kolmogorov-Smirnov test. Based on postprandial glucose response in our previous study (Numao et al. 2013), sample size was calculated to detect a large effect (Cohen's $d=0.98$ ). A sample size of 11 would be required to have approximately $80 \%$ power to detect a large effect at 0.05 significance. Because GLP-1, TG, and $\beta$-HBA concentrations did not assume a normal distribution, these variables were log-transformed. Two-way repeated-measured analysis of variance (ANOVA) (trial $\times$ time) was used to compare changes in body mass, body mass index (BMI), \%fat, FM, FFM, plasma glucose, serum insulin, HOMA-IR, FFA, $\beta$-HBA, TC, HDLC, LDLC, TG, GIP, GLP-1, ALT, AST, ICAM-1, and VCAM-1 among trials over time. When a significant interaction was observed, Bonferroni post-hoc analysis was performed to determine differences among trials at a specific point in time. One-way repeated-measures ANOVA with Bonferroni post-hoc analysis was used to detect differences in the iAUC of the metabolites, insulin sensitivity indices, and insulin secretion indices among trials. The effect size was calculated using Cohen's $d$ (small: $\geq 0.20$, medium: $\geq 0.50$, or large: $\geq 0.80$ ) for comparison of the iAUC of the metabolites, insulin sensitivity indices, 
and insulin secretion indices among trials. Statistical analyses were performed using SPSS software version 21 (IBM Corporation, NY, USA). Statistical significance was set at $P<$ 0.05 .

\section{Results}

\subsection{Characteristics}

Body mass, BMI, \%fat, FM, and FFM remained unchanged in the C, ICIF, and LCHF trials (Table 2).

\subsection{Concentrations of fasting metabolites}

Fasting metabolite concentrations were shown in Table 3. Fasting plasma glucose decreased significantly in the C, ICIF, and LCHF trials; however, this trend did not differ among the 3 trials. TG concentration decreased significantly in the LCHF trial but remained unchanged in the $\mathrm{C}$ and ICIF trials. FFA and $\beta$-HBA concentrations increased significantly in the LCHF trial, but remained unchanged in the $\mathrm{C}$ and ICIF trials. HOMA-IR, TC, HDLC, LDLC, ALT, AST, ICAM-1, and VCAM-1 remained unchanged after the 3 trials.

\subsection{Concentrations of fasting hormones}

Fasting hormone concentrations were shown in Table 3. Fasting serum insulin concentrations 
decreased significantly in the C, ICIF, and LCHF trials (Table3), but the change did not differ among the 3 trials. GIP and GLP-1 remained unchanged after the 3 trials.

\subsection{Metabolite concentrations and iAUC during MTT}

The changes of the metabolite concentrations during MMT are shown in Fig. 1-3, respectively. Moreover, the iAUC of the metabolite concentrations during MMT are shown in Table 4. The iAUC of plasma glucose concentration was significantly $56 \%$ higher in the LCHF trial than in the $\mathrm{C}$ trial $(P=0.009$; effect size: 1.77$)$ but did not significantly differ between the ICIF and C trials. The iAUC of plasma GIP, TG, and FFA concentrations did not differ among the 3 trials. The iAUC of serum $\beta$-HBA concentration was significantly $1075 \%$ lower in the LCHF trial than in the $\mathrm{C}$ trial $(P=0.027$; effect size: 1.45$)$ but did not significantly differ between the ICIF and C trials. The iAUC of serum ICAM-1 concentration did not differ among the 3 trails, whereas that of VCAM-1 concentration was significantly $344 \%$ higher in the LCHF trial than in the C trial $(P=0.044$; effect size: 1.29$)$ but did not significantly differ between the ICIF and C trials.

\subsection{Hormone concentrations and iAUC during MTT}

The changes of the hormone concentrations during MMT are shown in Fig. 4, Moreover, the iAUC of the hormone concentrations during MMT are shown in Table 4. The iAUC of serum 
insulin concentration did not differ significantly among the 3 trials. The iAUC of plasma

GLP-1 concentration was significantly $63 \%$ higher in the LCHF trial than in the $\mathrm{C}$ trial $(P=$ 0.014; effect size: 1.45) but did not differ significantly between the ICIF and C trials. Plasma GIP concentrations did not differ among the 3 trials.

\subsection{Insulin sensitivity and first-phase insulin release}

The Matsuda index and OGIS did not differ significantly among the C, ICIF, and LCHF trials

(Table 5). The insulinogenic index was significantly lower in the LCHF trial than in the C trial $(P=0.044$; effect size: 1.26$)$ but did not significantly differ between the ICIF and C trials. The Stumvoll index tended to be lower in the LCHF trial than in the C trial $(P=0.20$;

effect size: 0.83 ).

\section{Discussion}

To the best of our knowledge, the present study is the first to investigate the dose-response relationship of short-term dietary carbohydrate and fat intake with aggravation of postprandial glucose metabolism and circulating adhesion molecules in healthy males. Our data demonstrated that compared with short-term intake of C, short-term intake of LCHF excessively increased postprandial glucose and VCAM-1 concentrations and attenuated 
first-phase insulin release. Intake of ICIF and C had similar effects on postprandial glucose metabolism and circulating adhesion molecules in healthy males. These findings suggest that a eucaloric diet containing a high percentage of fats may aggravate postprandial glucose metabolism and endothelial function in healthy males.

Our results are in agreement with the results of previous studies using OGTT that reported that short-term (2-3 days) intake of eucaloric LCHF (approximately 5\%-20\% carbohydrates and $70 \%-73 \%$ fats) excessively increased postprandial glucose concentration (Numao et al. 2012, 2013; Pehleman et al. 2005) and suppressed first-phase insulin release (Numao et al. 2012,2013 ) compared with a control diet (approximately 51\%-62\% carbohydrates and 29\%$30 \%$ fats). Moreover, a previous study using intravenous glucose tolerance test demonstrated that short-term (approximately 3 days) intake of eucaloric LCHF (approximately 0\% carbohydrates and $85 \%$ fats) aggravated glucose tolerance compared with a control diet (approximately 50\% carbohydrates and 35\% fats) (Johnson et al. 2006). In contrast, although the response of postprandial glucose metabolism was not directly observed, previous studies using an intravenous glucose tolerance test or euglycemic hyperinsulinemic clamp (Branis et al. 2015) showed that short-term (5-7 days) intake of eucaloric ICIF (30\% carbohydrates and $50 \%-55 \%$ fats $)$ did not decrease insulin sensitivity compared with a control diet $(50 \%-55 \%$ 
carbohydrates and $30 \%$ fats). Therefore, it is possible that short-term intake of a eucaloric diet containing $>70 \%$ fats may aggravate postprandial glucose metabolism, whereas a eucaloric diet containing $<50 \%$ fats may have little effect on the aggravation of postprandial glucose metabolism.

The direct reason for the excessive increase in postprandial glucose concentration after LCHF may be a decrease in postprandial glucose disposal by the suppression of first-phase insulin release. This suppression of first-phase insulin release leads to the reduction of peripheral and hepatic glucose uptake and suppression of hepatic glucose production in the postprandial state. Of note, LCHF was found to reduce the ability of insulin to suppress endogenous glucose production (Bisschop et al. 2001). Therefore, in the present study, the reduction in the suppression of hepatic glucose production may explain the excessive increase in postprandial glucose concentration after LCHF. On the other hand, the decrease in first-phase insulin release after LCHF appears to be due to prolonged exposure to a high concentration of FFA in blood. The effect of serum FFA on insulin secretion is controversial. Nevertheless, prolonged elevation of serum FFA concentration leads to impaired insulin secretion (Oprescu et al. 2007). In the present study, fasting FFA and $\beta$-HBA concentrations increased after a 3 -day intake of LCHF and were higher than those after a 3-day intake of C. In contrast, fasting FFA 
and $\beta$-HBA concentrations remained unchanged after a 3-day intake of ICIF and did not differ from that those after a 3-day intake of C. These results indicate that a 3-d intake of LCHF induced prolonged elevation of serum FFA concentration, which may decrease insulin secretion and consequently increase postprandial glucose concentration excessively.

The iAUC of circulating VCAM-1 concentration was higher after LCHF than after C. To the best of our best knowledge, this is the first evidence of an increase in postprandial VCAM-1 concentration after short-term intake of LCHF, which suggests that even an acute excessive increase in postprandial glucose concentration may cause endothelial activation. In healthy individuals, circulating soluble adhesion molecules have been reported to increase by even acute increases in glucose concentration under normal range of postprandial glucose concentration (peak postprandial glucose concentration: approximately $8 \mathrm{mmol} / \mathrm{l})($ Ceriello et al. 2004; Derosa et al. 2010). Moreover, the increase in circulating soluble adhesion molecules during acute hyperglycemia has been reported to be regulated by endogenous insulin secretion (Marfella et al. 2000; Perkins et al. 2015). Therefore, the decrease in first-phase insulin secretion as well as the excessive increase in postprandial glucose concentration after LCHF may have contributed to the increase in circulating VCAM-1 concentration. Unlike the iAUC of circulating VCAM-1 concentration, that of ICAM-1 
concentration did not differ among the 3 diets. This suggests that circulating VCAM-1 and ICAM-1 concentrations were regulated by different pathways. The underlying mechanism is potentially mediated via advanced glycation end products (AGEs). AGEs form and increase in hyperglycemic environments (Brownlee 1995) and following the 2-h standard meal test (Beisswenger et al. 2001). The increased postprandial AGEs (methylglyoxal and 3-deoxyglucosone as highly reactive AGE precursors) are highly correlated with postprandial glycemic excursions (Beisswenger et al. 2001), suggesting that an excessive increase in postprandial glucose concentration after meal ingestion promotes the production of AGEs. Moreover, increased postprandial AGEs may interact with the endothelial receptors of AGEs and up-regulate the transcription factor NF-кB (Bierhaus et al. 1997; Schmidt et al. 1994), which may transcribe VCAM-1 and ICAM-1 genes (Basta et al. 2004). Although there is no evidence of an association between postprandial AGE and activation of NF- $\kappa \mathrm{B}, \mathrm{NF}-\kappa \mathrm{B}$ can be activated in postprandial state (up to 2 hours after meal ingestion) (Aljada et al. 2004). Furthermore, increased postprandial AGEs affect the postprandial serum VCAM-1 and ICAM-1 concentrations (Negrean et al. 2007). Considering these facts, it is possible that increased AGEs, due to an excessive increase in postprandial glucose concentration, are involved in the postprandial response of VCAM-1 and ICAM-1 concentrations, mediated by 
the activation of NF- $\kappa B$. AGEs selectively enhance endothelial VCAM-1 expression, whereas their effect on ICAM-1 has not been observed in vitro (Schmidt et al.1995). Although ingestion of an AGEs-rich meal acutely increases both postprandial serum VCAM-1 and ICAM-1 concentrations, the postprandial response in serum VCAM-1 appears to be remarkably greater than that in serum ICAM-1 (Negrean et al. 2007). Therefore, it is speculated that the increase in AGEs via excessive increase in postprandial glucose concentration after a short-term intake of LCHF is involved in the selective increase in the postprandial serum VCAM-1 concentration.

The greater increase in GLP-1 concentration after LCHF was in agreement with the results of previous studies (Numao et al. 2012, 2013). This increase in GLP-1 concentration after a 3-day intake of LCHF may represent a physiological response to enhance insulin secretion for replenishing deficient glucose in various tissues, such as the liver and skeletal muscle. The potential mechanism for this increase in GLP-1 may be explained by an increase in bile acids, which may specifically increase GLP-1 concentration. Experimental administration of bile acids was shown to stimulate GLP-1 secretion via L-cells and consequently increase GLP-1 concentration during fasting and postprandial states in healthy humans (Meyer-Gerspach et al. 2013; Murakami et al. 2013; Wu et al. 2013); however, the effect of 
bile acids on GIP concentration remains unclear. Moreover, the intake of high-fat diet elevates fecal concentration of bile acids (Cummings et al. 1978), suggesting a perfusion of a high concentration of bile acids into L-cells localized in the intestine after the intake of high-fat diet. Thus, the increased bile acids induced by the intake of LCHF may selectively promote GLP-1 secretion.

There are several limitations in the present study. First, the participants were young healthy males. Therefore, our findings may not be applicable to older adults or those with impaired glucose tolerance and diabetes. However, it is possible that alterations in postprandial glucose metabolism and circulating soluble adhesion molecules after a short-term intake of LCHF would be greater in such individuals than in young healthy males, because perturbations in postprandial glucose concentration and circulating soluble adhesion molecules are likely to be exaggerated in such individuals. Second, the sample size $(n=7)$ was smaller than the preliminarily estimated sample size $(n=11)$. Nevertheless, the significant differences observed in the present study were accompanied by a high effect size (Cohen's $d>1.26$ ) with adequate statistical power $(>79 \%)$. Therefore, our findings on the effects of LCHF on postprandial glucose metabolism and circulating soluble adhesion molecules are reliable.

In conclusion, we observed that short-term intake of eucaloric LCHF aggravated the response 
of postprandial glucose metabolism and circulating VCAM-1 concentration in healthy males, whereas eucaloric ICIF had little effects. Thus, short-term intake of a eucaloric diet containing more than $70 \%$ fats may contribute to the development of impaired glucose tolerance, diabetes, and arteriosclerosis in healthy males.

\section{Conflicts of interest}

None of the authors had a conflict of interest.

\section{Acknowledgment}

The authors thank all the participants of the present study. The present study was supported by Waseda University Individual Research Allowance. 


\section{References}

Aljada, A., Mohanty, P., Ghanim, H., Abdo, T., Tripathy, D., Chaudhuri, A., et al. 2004.

Increase in intranuclear nuclear factor $\mathrm{\kappa B}$ and decrease in inhibitor $\kappa \mathrm{B}$ in mononuclear cells

after a mixed meal: evidence for a proinflammatory effect. Am. J. Clin. Nutr. 79: 682-690.

PMID:15051615.

Anderson, A.S., Haynie, K.R., McMillan, R.P., Osterberg, K.L., Boutagy, N.E., Frisard, M.I., et al. 2015. Early skeletal muscle adaptations to short-term high-fat diet in humans before changes in insulin sensitivity. Obesity. 23: 720-724. doi:10.1002/oby.21031.

PMID:25820254.

Basta, G., Schmidt, A.M., and De Caterina, R. 2004. Advanced glycation end products and vascular inflammation: implications for accelerated atherosclerosis in diabetes. Cardiovasc.

Res. 63: 582-592. PMID:15306213.

Beisswenger, P. J., Howell, S. K., O'Dell, R. M., Wood, M. E., Touchette, A. D., and Szwergold, B. S. 2001. $\alpha$-Dicarbonyls increase in the postprandial period and reflect the degree of hyperglycemia. Diabetes care. 24: 726-732. PMID:11315838.

Bierhaus, A., Illmer, T., Kasper, M., Luther, T., Quehenberger, P., Tritschler, H., et al. 1997. Advanced glycation end product (AGE)-mediated induction of tissue factor in cultured 
endothelial cells is dependent on RAGE. Circulation. 96:2262-2271. PMID:9337199.

Bisschop, P.H., de Metz, J., Ackermans, M.T., Endert, E., Pijl, H., Kuipers, F., et al. 2001.

Dietary fat content alters insulin-mediated glucose metabolism in healthy men. Am. J. Clin.

Nutr. 73: 554-559. PMID:11237931.

Branis, N.M., Etesami, M., Walker, R.W., Berk, E.S., and Albu, J.B. 2015. Effect of a 1-week, eucaloric, moderately high-fat diet on peripheral insulin sensitivity in healthy premenopausal women. BMJ Open Diabetes Res. Care. 3: e000100. doi:10.1136/bmjdrc-2015-000100. PMID:26203360.

Brownlee, M. 1995. Advanced protein glycosylation in diabetes and aging. Annu. Rev. Med.

46: 223-234. PMID:7598459.

Cavalot, F., Pagliarino, A., Valle, M., Di Martino, L., Bonomo, K., Massucco, P., et al. 2011. Postprandial Blood Glucose Predicts Cardiovascular Events and All-Cause Mortality in Type 2 Diabetes in a 14-Year Follow-Up Lessons from the San Luigi Gonzaga Diabetes Study. Diabetes Care. 34: 2237-2243. doi: 10.2337/dc10-2414. PMID:21949221.

Ceriello, A., Quagliaro, L., Piconi, L., Assaloni, R., Da Ros, R., Maier, A., et al. 2004. Effect of postprandial hypertriglyceridemia and hyperglycemia on circulating adhesion molecules and oxidative stress generation and the possible role of simvastatin treatment. Diabetes. 53: 
701-710. PMID:14988255.

Cowie, C.C., Rust, K.F., Ford, E.S., Eberhardt, M.S., Byrd-Holt, D.D., Li, C., et al. 2009.

Full accounting of diabetes and pre-diabetes in the U.S. population in 1988-1994 and 2005-2006. Diabetes Care. 32: 287-294. doi:10.2337/dc08-1296. PMID:19017771.

Cummings, J.H., Wiggins, H.S., Jenkins, D.J., Houston, H., Jivraj, T., Drasar, B.S., et al. 1978. Influence of diets high and low in animal fat on bowel habit, gastrointestinal transit time, fecal microflora, bile acid, and fat excretion. J. Clin. Invest. 61:953-963. PMID:659584.

The DECODE group. 1999. Glucose tolerance and mortality: comparison of WHO and American Diabetes Association diagnostic criteria. The DECODE study group. European Diabetes Epidemiology Group. Diabetes Epidemiology: Collaborative analysis Of Diagnostic criteria in Europe. Lancet. 354: 617-621. PMID:10466661.

The DECODE group. 2001. Glucose tolerance and cardiovascular mortality: comparison of fasting and 2-hour diagnostic criteria. Arch. Intern. Med. 161: 397-405. PMID:11176766.

Derosa, G., D'Angelo, A., Salvadeo, S.A., Ferrari, I., Fogari, E., Gravina, A., et al. 2010. Oral glucose tolerance test effects on endothelial inflammation markers in healthy subjects and diabetic patients. Horm. Metab. Res. 42: 8-13. doi:10.1055/s-0029-1237728. PMID:19735057. 
Esposito, K., Nappo, F., Marfella, R., Giugliano, G., Giugliano, F., Ciotola, M., et al. 2002.

Inflammatory cytokine concentrations are acutely increased by hyperglycemia in humans:

role of oxidative stress. Circulation. 106: 2067-2072. PMID:12379575.

Friedewald, W.T., Levy, R.I., and Fredrickson, D.S. 1972. Estimation of the concentration of low-density lipoprotein cholesterol in plasma, without use of the preparative ultracentrifuge.

Clin. Chem. 18: 499-502. PMID:4337382.

Henninger, J., Hammarstedt, A., Rawshani, A., and Eliasson, B. 2015. Metabolic predictors of impaired glucose tolerance and type 2 diabetes in a predisposed population--A prospective cohort study. BMC Endocr Disord. 15: 51. doi: 10.1186/s12902-015-0048-8. PMID:26407933.

Johnson, N.A., Stannard, S.R., Rowlands, D.S., Chapman, P.G., Thompson, C.H., O'Connor, H., et al. 2006. Effect of short-term starvation versus high-fat diet on intramyocellular triglyceride accumulation and insulin resistance in physically fit men. Exp. Physiol. 91: 693-703. PMID:16627573.

Kawano, H., Motoyama, T., Hirashima, O., Hirai, N., Miyao, Y., Sakamoto, T., et al. 1999. Hyperglycemia rapidly suppresses flow-mediated endothelium-dependent vasodilation of brachial artery. J. Am. Coll. Cardiol. 34: 146-154. PMID:10400004. 
Mah, E., and Bruno, R.S. 2012. Postprandial hyperglycemia on vascular endothelial function: mechanisms and consequences. Nutr. Res. 32: 727-740. doi:10.1016/j.nutres.2012.08.002.

PMID:23146769.

Marfella, R., Esposito, K., Giunta, R., Coppola, G., De Angelis, L., Farzati, B., et al. 2000.

Circulating adhesion molecules in humans: role of hyperglycemia and hyperinsulinemia.

Circulation. 101: 2247-2251. PMID:10811590.

Mari, A., Pacini, G., Murphy, E., Ludvik, B., and Nolan, J.J. 2001. A model-based method for assessing insulin sensitivity from the oral glucose tolerance test. Diabetes Care. 24: 539-548.

PMID:11289482.

Matsuda, M., and DeFronzo, R.A. 1999. Insulin sensitivity indices obtained from oral glucose tolerance testing: comparison with the euglycemic insulin clamp. Diabetes Care. 22:

1462-1470. PMID:10480510.

Meyer-Gerspach, A.C., Steinert, R.E., Keller, S., Malarski, A., Schulte, F.H., and Beglinger, C. 2013. Effects of chenodeoxycholic acid on the secretion of gut peptides and fibroblast growth factors in healthy humans. J. Clin. Endocrinol. Metab. 98: 3351-3358. doi:10.1210/jc.2012-4109. PMID:23783097.

Ministry of Health, Labour and Walefare. 2010. Dietary reference intakes for Japanese, 2010. 
Daiichi Shuppan, Tokyo. (in Japanese).

Murakami, M., Une, N., Nishizawa, M., Suzuki, S., Ito, H., and Horiuchi, T. 2013. Incretin secretion stimulated by ursodeoxycholic acid in healthy subjects. Springer Plus. 2:20. doi:10.1186/2193-1801-2-20. PMID:23450079.

Nakagami, T., and DECODA Study Group. 2004. Hyperglycaemia and mortality from all causes and from cardiovascular disease in five populations of Asian origin. Diabetologia. 47:

385-394. PMID:14985967.

Negrean, M., Stirban, A., Stratmann, B., Gawlowski, T., Horstmann, T., Götting, C., et al. 2007. Effects of low- and high-advanced glycation endproduct meals on macro- and microvascular endothelial function and oxidative stress in patients with type 2 diabetes mellitus. Am. J. Clin. Nutr. 85: 1236-1243. PMID:17490958.

Numao, S., Kawano, H., Endo, N., Yamada, Y., Konishi, M., Takahashi, M., et al. 2012. Short-term low carbohydrate/high-fat diet intake increases postprandial plasma glucose and glucagon-like peptide-1 levels during an oral glucose tolerance test in healthy men. Eur. J.

Clin. Nutr. 66: 926-931. doi:10.1038/ejcn.2012.58. PMID:22669333.

Numao, S., Kawano, H., Endo, N., Yamada, Y., Konishi, M., Takahashi, M., et al. 2013. Effects of a single bout of aerobic exercise on short-term low-carbohydrate/high-fat 
intake-induced postprandial glucose metabolism during an oral glucose tolerance test. Metabolism. 62: 1406-1415. doi:10.1016/j.metabol.2013.05.005. PMID:23764436.

Oprescu, A.I., Bikopoulos, G., Naassan, A., Allister, E.M., Tang, C., Park, E., et al. 2007. Free fatty acid-induced reduction in glucose-stimulated insulin secretion: evidence for a role of oxidative stress in vitro and in vivo. Diabetes. 56: 2927-2937. PMID:17717282.

Pehleman, T.L., Peters, S.J., Heigenhauser, G.J., and Spriet, L.L. 2005. Enzymatic regulation of glucose disposal in human skeletal muscle after a high-fat, low-carbohydrate diet. J. Appl.

Physiol. 98: 100-107. PMID:15310747.

Perkins, J.M., Joy, N.G., Tate, D.B., and Davis, S.N. 2015. Acute effects of hyperinsulinemia and hyperglycemia on vascular inflammatory biomarkers and endothelial function in overweight and obese humans. Am. J. Physiol. Endocrinol. Metab. 309: E168-176. doi:10.1152/ajpendo.00064.2015. PMID:26015434.

Sakamoto, T., Ogawa, H., Kawano, H., Hirai, N., Miyamoto, S., Takazoe, K., et al. 2000. Rapid change of platelet aggregability in acute hyperglycemia. Detection by a novel laser-light scattering method. Thromb. Haemost. 83: 475-479. PMID:10744156.

Schmidt, A.M., Hasu, M., Popov, D., Zhang, J.H., Chen, J., Yan, S.D., et al. 1994. Receptor for advanced glycation end products (AGEs) has a central role in vessel wall interactions and 
gene activation in response to circulating AGE proteins. Proc. Natl. Acad. Sci. U S A. 91:

8807-8811. PMID:8090728.

Schmidt, A.M., Hori, O., Chen, J.X., Li, J.F., Crandall, J., Zhang, J., et al. 1995. Advanced glycation endproducts interacting with their endothelial receptor induce expression of vascular cell adhesion molecule-1 (VCAM-1) in cultured human endothelial cells and in mice. A potential mechanism for the accelerated vasculopathy of diabetes. J. Clin. Invest. 96: 1395-1403. PMID:7544803.

Soriguer, F., Goday, A., Bosch-Comas, A., Bordiú, E., Calle-Pascual, A., Carmena, R., et al. 2012. Prevalence of diabetes mellitus and impaired glucose regulation in Spain: the Di@bet.es $\quad$ Study. $\quad$ Diabetologia. $\quad$ 55: $\quad 88-93 . \quad$ doi:10.1007/s00125-011-2336-9. PMID:21987347.

Stumvoll, M., Mitrakou, A., Pimenta, W., Jenssen, T., Yki-Jarvinen, H., Van Haeften, T., et al. 2000. Use of the oral glucose tolerance test to assess insulin release and insulin sensitivity.

Diabetes Care. 23: 295-301. PMID:10868854.

Williams, S.B., Goldfine, A.B., Timimi, F.K., Ting, H.H., Roddy, M.A., Simonson, D.C., et al. 1998. Acute hyperglycemia attenuates endothelium-dependent vasodilation in humans in vivo.

Circulation. 97: 1695-1701. PMID:9591763. 
Wu, T., Bound, M.J., Standfield, S.D., Gedulin, B., Jones, K.L., Horowitz, M., et al. 2013.

Effects of rectal administration of taurocholic acid on glucagon-like peptide-1 and peptide

YY secretion in healthy humans. Diabetes. Obes. Metab. 15: 474-477.

doi:10.1111/dom.12043. PMID:23181598. 
Table 1. Nutrient content of control diet, intermediate-carbohydrate/intermediate-fat diet and low-carbohydrate/high-fat diet.

\begin{tabular}{|c|c|c|c|c|c|c|c|c|c|}
\hline \multirow[b]{3}{*}{ Energy (kcal) } & \multicolumn{3}{|c|}{$\mathrm{C}$} & \multicolumn{3}{|c|}{ ICIF } & \multicolumn{3}{|c|}{$\mathrm{LCHF}$} \\
\hline & Value & \multicolumn{2}{|c|}{ Range } & Value & \multicolumn{2}{|c|}{ Range } & Value & \multicolumn{2}{|c|}{ Range } \\
\hline & $2975 \pm 65$ & 2739 & -3102 & $2984 \pm 64$ & 2752 & -3109 & $2946 \pm 79$ & 2722 & -3254 \\
\hline Protein $(g)$ & $75 \pm 1$ & 72 & -76 & $77 \pm 2$ & 70 & -81 & $73 \pm 1$ & 70 & -76 \\
\hline Fat $(\mathrm{g}) *$ & $93 \pm 3$ & 83 & -99 & $169 \pm 3$ & 160 & -173 & $228 \pm 8$ & 207 & -256 \\
\hline Carbohydrate $(\mathrm{g}) *$ & $459 \pm 9$ & 426 & -476 & $291 \pm 8$ & 259 & -306 & $151 \pm 3$ & 145 & -163 \\
\hline \multicolumn{10}{|l|}{ Percentage to energy } \\
\hline Protein $(\%)$ & $10.0 \pm 0.1$ & 9.8 & -10.5 & $10.3 \pm 0.1$ & 9.9 & -10.4 & $10.0 \pm 0.1$ & 9.3 & -10.3 \\
\hline Fat $(\%) *$ & $28.2 \pm 0.3$ & 27.3 & -28.8 & $50.8 \pm 0.4$ & 50.2 & -52.2 & $69.5 \pm 0.5$ & 67.6 & -70.8 \\
\hline Carbohydrate $(\%) *$ & $61.8 \pm 0.2$ & 61.4 & -62.6 & $38.9 \pm 0.3$ & 37.7 & -39.4 & $20.5 \pm 0.4$ & 19.4 & -22.2 \\
\hline
\end{tabular}

Data represent means \pm SE. C, control diet; ICIF, intermediate-carbohydrate/intermediate-fat diet; LCHF, low-carbohydrate/high-fat diet. significantly different among three diet. 
Table 2. Change in subject characteristics during 3-day diet intervention.

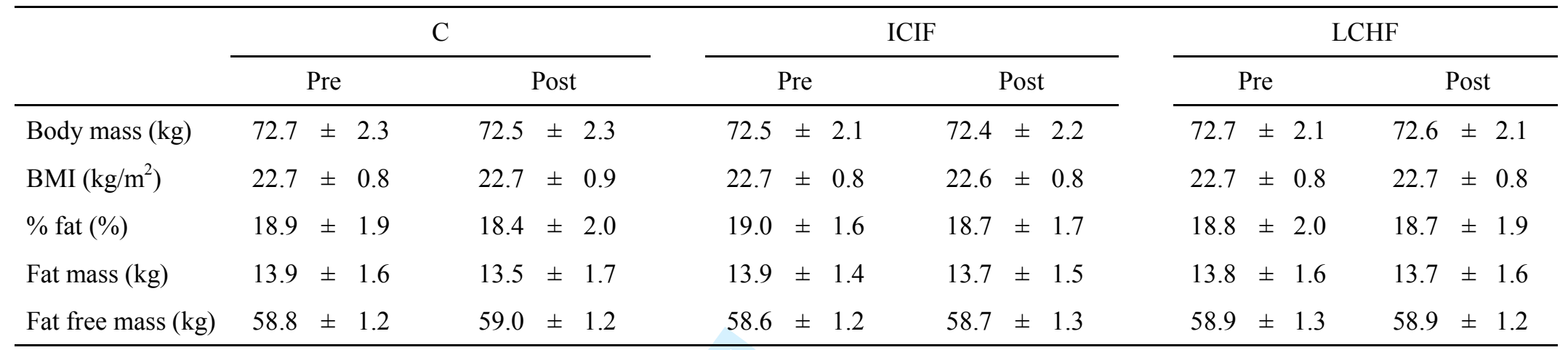

Data represent means \pm SE. C, control diet; ICIF, intermediate-carbohydrate/intermediate-fat diet; LCHF, low-carbohydrate/high-fat diet; BMI, body mass index. 
Table 3. Change in fasting blood metabolite and hormone concentration during 3-day intervention.

\begin{tabular}{|c|c|c|c|c|c|c|c|c|c|c|c|c|c|c|c|c|c|c|}
\hline \multirow{2}{*}{ Metabolites } & \multicolumn{6}{|c|}{$\mathrm{C}$} & \multicolumn{6}{|c|}{ ICIF } & \multicolumn{6}{|c|}{ LCHF } \\
\hline & \multicolumn{3}{|c|}{ Pre } & \multicolumn{3}{|c|}{ Post } & \multicolumn{3}{|c|}{ Pre } & \multicolumn{3}{|c|}{ Post } & \multicolumn{3}{|c|}{ Pre } & \multicolumn{3}{|c|}{ Post } \\
\hline Glucose (mmol/1) & 5.0 & \pm & 0.1 & 4.9 & \pm & $0.1 *$ & 5.2 & \pm & 0.1 & 4.9 & \pm & $0.2 *$ & 5.1 & \pm & 0.1 & 4.7 & \pm & $0.1 *$ \\
\hline $\mathrm{TC}(\mathrm{mmol} / \mathrm{l})$ & 4.49 & \pm & 0.37 & 4.58 & \pm & 0.35 & 4.53 & \pm & 0.39 & 4.50 & \pm & 0.32 & 4.55 & \pm & 0.39 & 4.51 & \pm & 0.33 \\
\hline HDLC (mmol/1) & 1.45 & \pm & 0.15 & 1.43 & \pm & 0.14 & 1.42 & \pm & 0.14 & 1.48 & \pm & 0.14 & 1.49 & \pm & 0.13 & 1.55 & \pm & 0.11 \\
\hline LDLC (mmol/l) & 2.41 & \pm & 0.19 & 2.44 & \pm & 0.20 & 2.51 & \pm & 0.29 & 2.47 & \pm & 0.21 & 2.32 & \pm & 0.18 & 2.58 & \pm & 0.21 \\
\hline $\mathrm{FFA}(\mathrm{mmol} / \mathrm{l})$ & 0.29 & \pm & 0.05 & 0.36 & \pm & 0.02 & 0.28 & \pm & 0.02 & 0.35 & \pm & 0.03 & 0.29 & \pm & 0.02 & 0.52 & \pm & $0.04 * \dagger$ \\
\hline $\mathrm{TG}(\mathrm{mmol} / \mathrm{l})$ & 1.39 & \pm & 0.33 & 1.56 & \pm & 0.53 & 1.30 & \pm & 0.32 & 1.21 & \pm & 0.29 & 1.63 & \pm & 0.46 & 0.83 & \pm & $0.21 * \dagger$ \\
\hline$\beta-\mathrm{HBA}(\mu \mathrm{mol} / 1)$ & 30 & \pm & 9 & 25 & \pm & 3 & 28 & \pm & 7 & 23 & \pm & 3 & 22 & \pm & 3 & 86 & \pm & $22 * \dagger$ \\
\hline $\operatorname{ALT}(\mathrm{U} / 1)$ & 20 & \pm & 3 & 20 & \pm & & 17 & \pm & 2 & 19 & \pm & 3 & 16 & \pm & 1 & 19 & & 2 \\
\hline AST (U/1) & 19 & \pm & 1 & 19 & \pm & 1 & 16 & \pm & 1 & 19 & \pm & 2 & 18 & \pm & 1 & 19 & \pm & 1 \\
\hline ICAM-1 (ng/ml) & 310.6 & \pm & 12.9 & 326.0 & \pm & 14.5 & 352.3 & \pm & 15.1 & 337.6 & \pm & 14.4 & 332.7 & \pm & 17.6 & 313.5 & \pm & 19.7 \\
\hline VCAM-1 (ng/ml) & 412.9 & \pm & 78.5 & 425.5 & \pm & 51.8 & 374.8 & \pm & 40.6 & 368.0 & \pm & 35.4 & 332.6 & \pm & 20.0 & 344.6 & \pm & 23.5 \\
\hline \multicolumn{19}{|l|}{ Hormones } \\
\hline Insulin (pmol/l) & 40.2 & \pm & 5.7 & 33.5 & \pm & $4.9 *$ & 37.5 & \pm & 6.2 & 33.1 & \pm & $7.8 *$ & 35.1 & \pm & 4.9 & 25.5 & \pm & $3.7 *$ \\
\hline GLP-1(pmol/1) & 5.1 & \pm & 0.3 & 5.0 & \pm & 0.2 & 5.1 & \pm & 0.2 & 5.1 & \pm & 0.2 & 5.4 & \pm & 0.3 & 5.3 & \pm & 0.3 \\
\hline GIP (pmol/l) & 44.4 & \pm & 12.0 & 41.2 & \pm & 6.0 & 40.3 & \pm & 8.8 & 43.2 & \pm & 9.6 & 45.7 & \pm & 7.5 & 38.0 & \pm & 3.8 \\
\hline \multicolumn{19}{|l|}{ Insulin sensitivity } \\
\hline HOMA-IR & 1.50 & \pm & 0.21 & 1.20 & \pm & 0.18 & 1.44 & \pm & 0.23 & 1.20 & \pm & 0.3 & 1.33 & \pm & 0.18 & 0.89 & \pm & 0.13 \\
\hline
\end{tabular}

Data represent means \pm SE. C, control diet; ICIF, intermediate-carbohydrate/intermediate-fat diet; LCHF, low-carbohydrate/high-fat diet; GLP-1, glucagon-like peptide 1; GIP, glucose-dependent insulinotropic polypeptide; FFA, free fatty acid; TG, triglycerides; TC, total cholesterol; HDLC, high-density lipoprotein cholesterol; LDLC, low-density lipoprotein cholesterol; $\beta$-HBA, $\beta$-hydroxybutyrate acid; ALT, alanine aminotransferase; AST, aspartate aminotransferase; ICAM-1, intercellular adhesion molecule-1; VCAM-1, vascular cell adhesion molecule-1. * significantly different from pre value within each diet $(\mathrm{P}<0.05)$. $\dagger$ significantly different from post value of $\mathrm{C}(\mathrm{P}<0.05)$. 
Table 4. Incremental area under curve during oral meal tolerance test.

\begin{tabular}{|c|c|c|c|c|c|c|c|c|c|c|c|c|c|}
\hline & \multirow{2}{*}{\multicolumn{3}{|c|}{$\mathrm{C}$}} & \multirow{2}{*}{\multicolumn{3}{|c|}{ ICIF }} & \multirow{2}{*}{\multicolumn{3}{|c|}{ LCHF }} & \multicolumn{2}{|c|}{ C vs ICIF } & \multicolumn{2}{|c|}{ C vs LCHF } \\
\hline & & & & & & & & & & $\mathrm{p}$ & ES & $\mathrm{p}$ & ES \\
\hline Glucose $(\mathrm{mmol} / 1 \cdot 2 \mathrm{~h})$ & 106 & \pm & 8 & 109 & \pm & 10 & 165 & \pm & $14 *$ & 1.000 & 0.07 & 0.009 & 1.77 \\
\hline $\mathrm{FFA}(\mathrm{mmol} / \mathrm{l} \cdot 2 \mathrm{~h})$ & -12.0 & \pm & 3.1 & -13.0 & \pm & 2.6 & -24.9 & \pm & 4.7 & 1.000 & 0.09 & 0.108 & 1.03 \\
\hline $\mathrm{TG}(\mathrm{mmol} / \mathrm{l} \cdot 2 \mathrm{~h})$ & 29 & \pm & 10 & 40 & \pm & 8 & 27 & \pm & 7 & 1.000 & 0.54 & 1.000 & 0.31 \\
\hline$\beta-\mathrm{HBA}(\mu \mathrm{mol} / 1 \cdot 2 \mathrm{~h})$ & 443 & \pm & 399 & 212 & \pm & 330 & -4323 & \pm & $1646^{*}$ & 0.947 & 0.43 & 0.027 & 1.45 \\
\hline ICAM-1 (ng/ml·2h) & -2630 & \pm & 1039 & -3356 & \pm & 874 & -3667 & \pm & 1266 & 1.000 & 0.16 & 1.000 & 0.36 \\
\hline VCAM-1(ng/ml-2h) & -2683 & \pm & 2039 & 797 & & 2596 & 6556 & \pm & $3388 *$ & 0.345 & 0.69 & 0.044 & 1.29 \\
\hline \multicolumn{14}{|l|}{ Hormones } \\
\hline Insulin $(\mathrm{pmol} / \mathrm{l} \cdot 2 \mathrm{~h})$ & 20557 & \pm & 2530 & 20468 & \pm & 4010 & 18385 & \pm & 2198 & 1.000 & 0.01 & 1.000 & 0.34 \\
\hline GLP-1(pmol/1·2h) & 107 & \pm & 29 & 67 & \pm & 26 & 174 & \pm & $24 *$ & 1.000 & 0.34 & 0.014 & 1.45 \\
\hline GIP (pmol/1·2h) & 32218 & \pm & 3081 & 30615 & \pm & 4637 & 33453 & \pm & 3585 & 1.000 & 0.29 & 1.000 & 0.23 \\
\hline
\end{tabular}

Data represent means \pm SE.C, control diet; ICIF, intermediate-carbohydrate/intermediate-fat diet; LCHF, low-carbohydrate/high-fat diet; ES, effect size; GLP-1, glucagon-like peptide 1; GIP, glucose-dependent insulinotropic polypeptide; FFA, free fatty acid; TG, triglycerides; $\beta$-HBA,

$\beta$-hydroxybutyrate acid; ICAM-1, intercellular adhesion molecule-1; VCAM-1, vascular cell adhesion molecule-1 * significantly different from the value of $\mathrm{C}(\mathrm{P}<0.05)$. 
Table 5. Insulin sensitivity and first-phase insulin release index after each intervention.

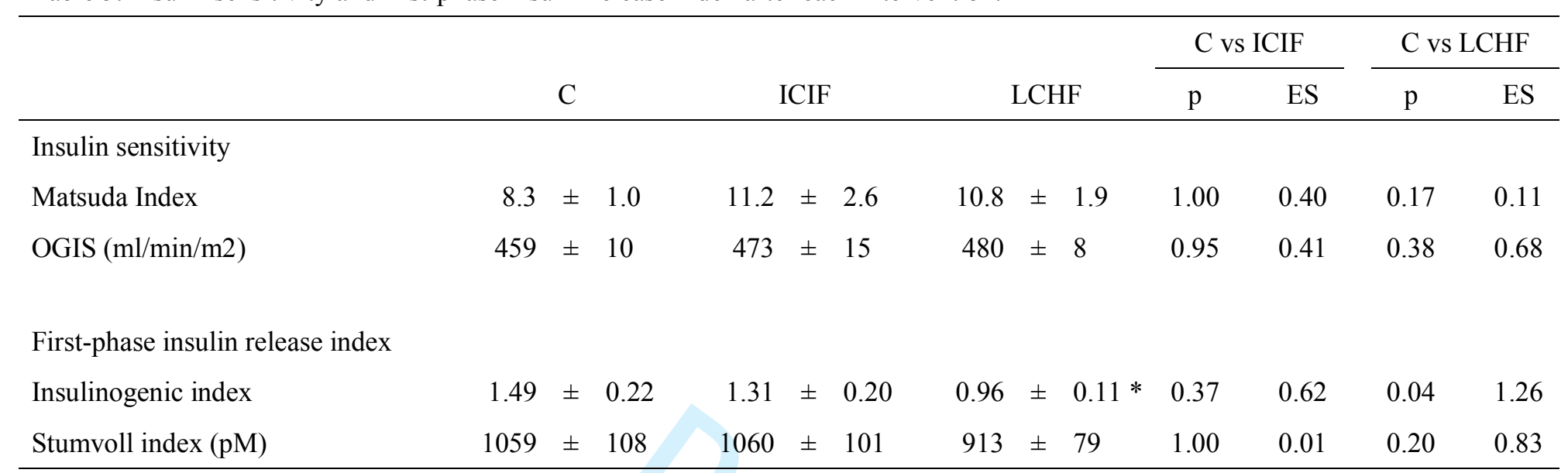

Data represent means \pm SE. C, control diet; ICIF, intermediate-carbohydrate/intermediate-fat diet; LCHF, low-carbhydrate/high-fat diet; OGIS, oral glucose insulin sensitivity. * significantly different from the value of $\mathrm{C}(P<0.05)$ 


\section{Figure captions}

\section{Fig. 1}

Changes in plasma glucose concentrations during MTT

C, control diet; ICIF, intermediate-carbohydrate/intermediate-fat diet; LCHF, low-carbohydrate/high-fat diet; MTT, meal tolerance test

\section{Fig. 2}

Changes in serum TG (upper), FFA (middle), and $\beta$-HBA acid (lower) concentrations during MTT

$\beta$-HBA, $\quad \beta$-hydroxybutyric acid; $\quad \mathrm{C}, \quad$ control diet; $\quad$ ICIF, intermediate-carbohydrate/intermediate-fat diet; FFA, free fatty acid; LCHF, low-carbohydrate/high-fat diet; MTT, meal tolerance test; TG, triglyceride

*significantly different between LCHF and C $(P<0.05)$

\section{Fig. 3}

Changes in serum ICAM-1 (upper) and VCAM-1 (lower) concentrations during MTT

C, control diet; ICAM-1, intercellular adhesion molecule-1; ICIF, 
intermediate-carbohydrate/intermediate-fat diet; LCHF, low-carbohydrate/high-fat diet; MTT, meal tolerance test; VCAM-1; vascular cell adhesion molecule-1

Fig. 4

Changes in serum insulin (upper), plasma GLP-1(middle) and GIP (lower) concentrations during MTT

C, control diet; GIP, glucose-dependent insulinotropic polypeptide; GLP-1, glucagon-like peptide-1; ICIF, intermediate-carbohydrate/intermediate-fat diet; LCHF, low-carbohydrate/high-fat diet; MTT, meal tolerance test

*significantly different between LCHF and C $(P<0.05)$ 
Figure 1

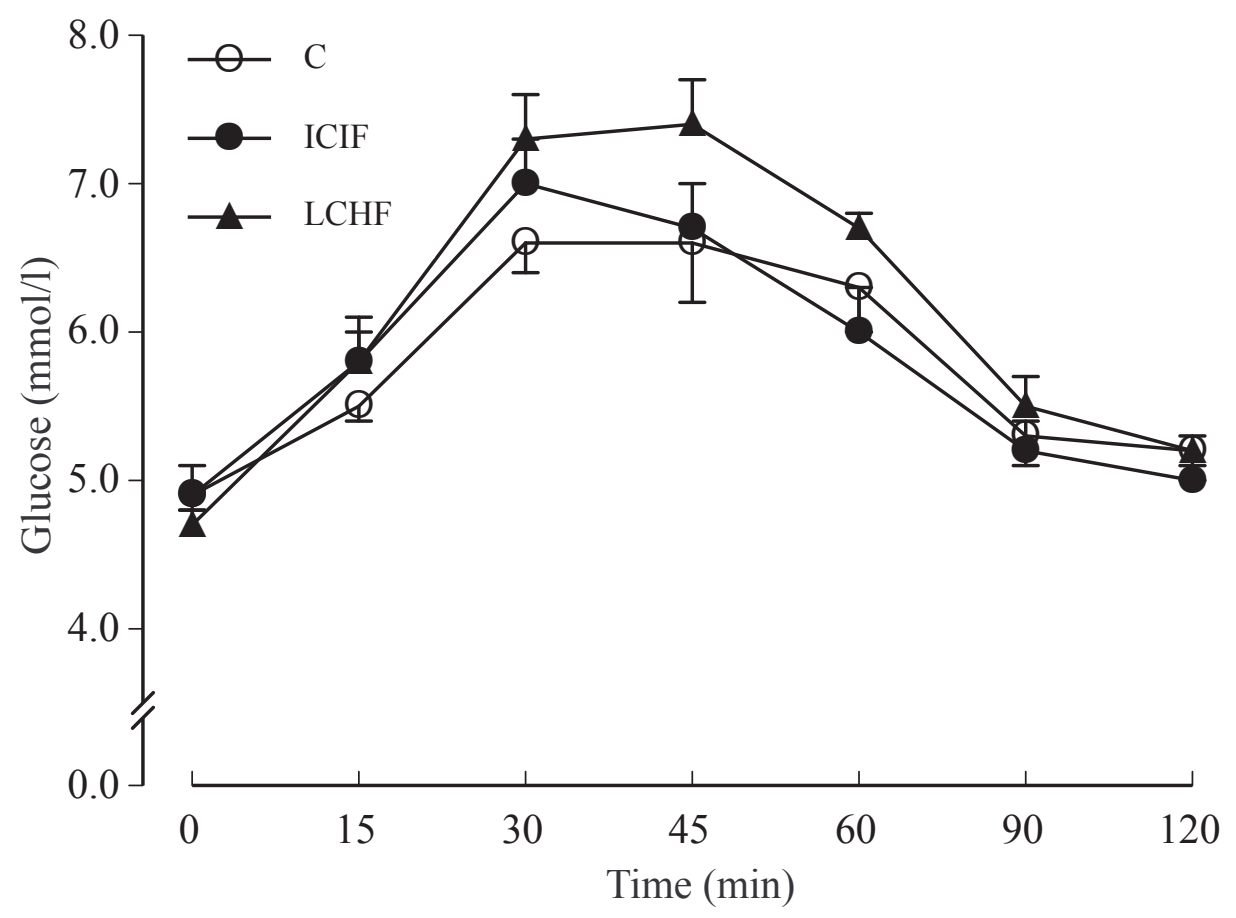



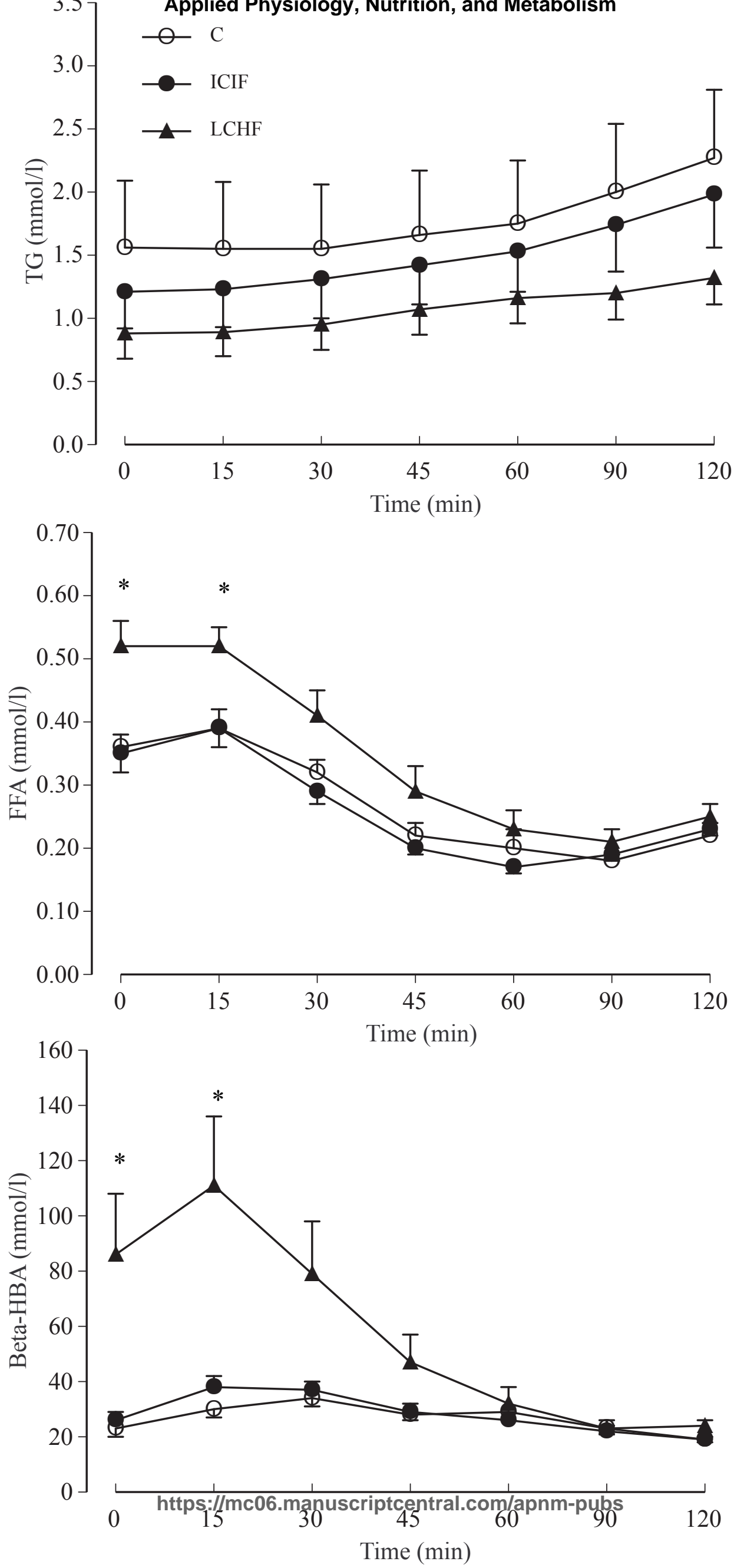

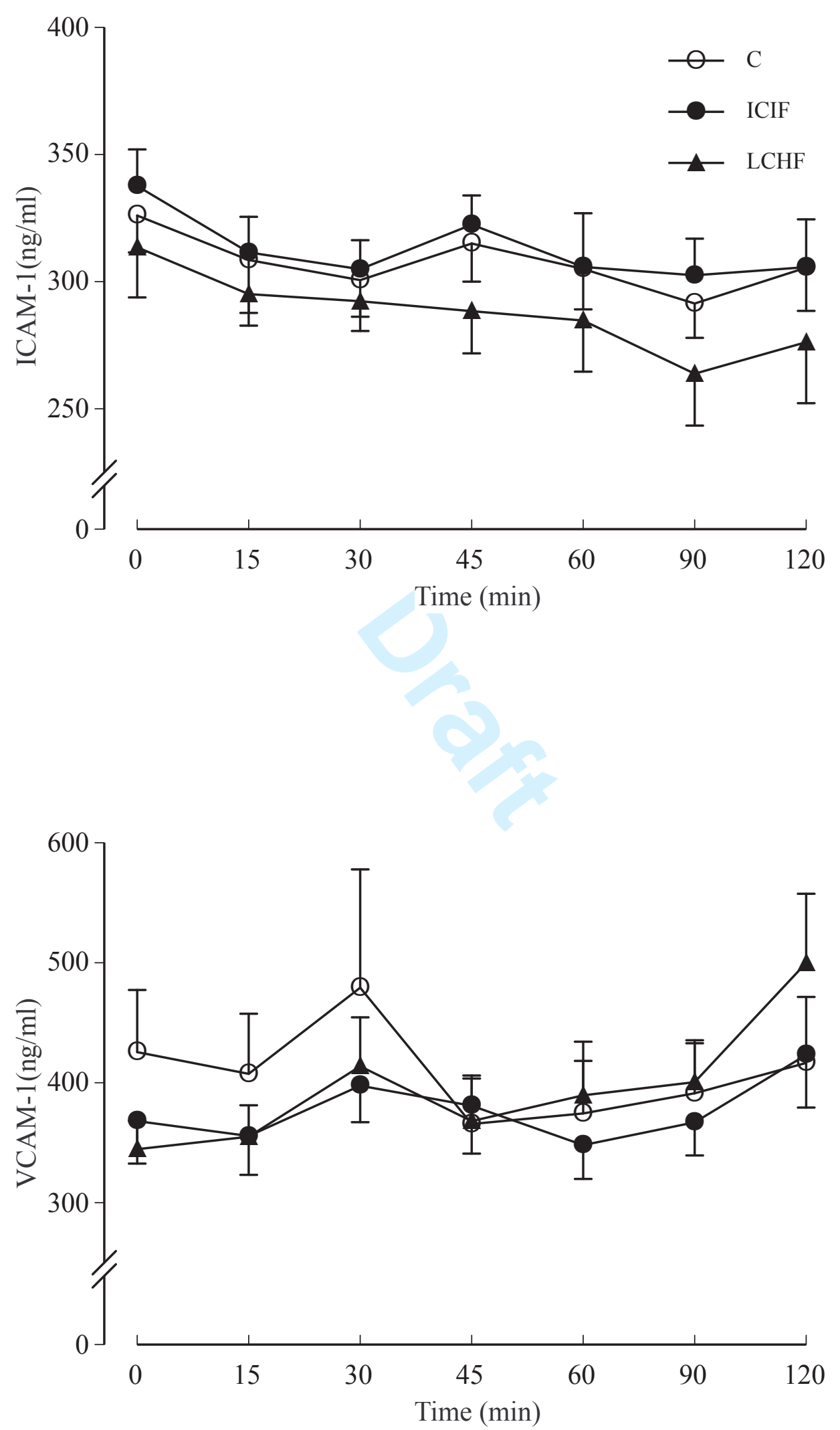

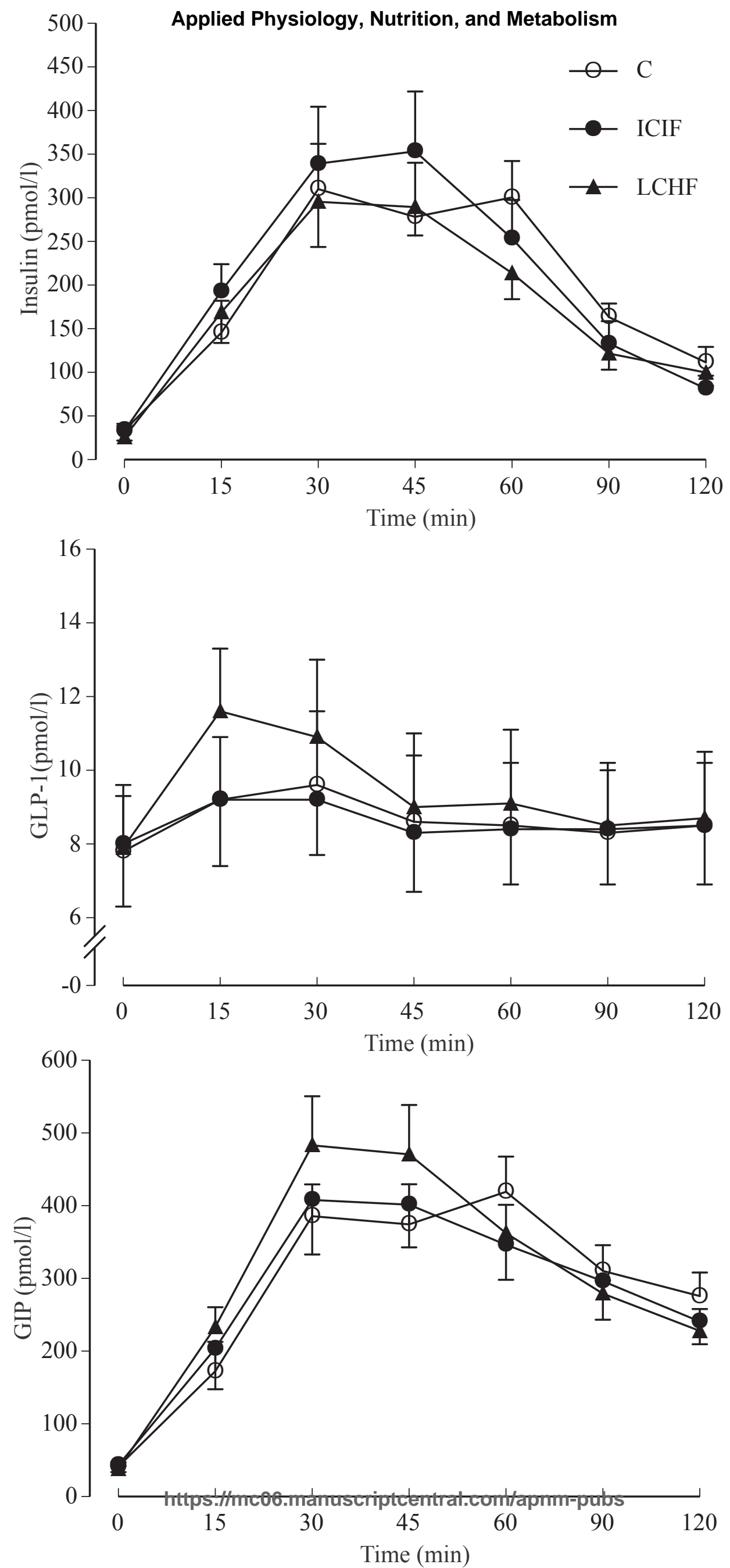